\title{
Internal field corrections in perpendicular columnar structured Alumite films
}

\author{
E.O. Samwel ${ }^{a}$, P.R. Bissell ${ }^{b}$ and J.C. Lodder ${ }^{a}$ \\ "MESA Research Institute, Unicersity of Twente, P.O. Box 217, 7500 AE Enschede, The Netherlands \\ ' Department of Physics and Astronomy, Lancashire Polytechnic, Preston PRI 2TQ, UK
}

Received 12 March 1992

\begin{abstract}
An investigation of the remanent and hysteretic properties of perpendicular media such as Alumite involves calculation of internal fields which requires the demagnetisation factor to be known. Demagnetisation effects have been calculated for Alumite, which is an ideal model material, by considering it as a hexagonal matrix of columns. This predicts that the demagnetising effects are less than that of a continuous film at saturation but increase as the magnetisation decreases.
\end{abstract}

\section{Introduction}

In the pursuit of high-density magnetic recording systems, longitudinal media are restricted by the demagnetisation factor associated with individual written bits, which requires reduction of the thickness of the magnetic layer as bit density is increased. An alternative approach is to use perpendicular media where the demagnetising factor of individual bits is no longer a limiting factor. There are many candidates for these systems such as $\mathrm{Co}-\mathrm{Cr}$ and other continuous films. $\mathrm{Ba}-\mathrm{Fe}$ particulate systems have current applications to longitudinal media but also have the potential for extension to perpendicular media. Alumite, although too complex a system for practical applications, is an ideal model material for perpendicular systems.

When analysing the behaviour of such systems, it is important to be able to relate macroscopic magnetic measurements on the film to the system behaviour experienced during the recording process. In longitudinal systems, because of the ab-

Correspondence to: E.O. Samwel, MESA Research Institute, University of Twente, P.O. Box 217, 7500 AE Enschede, The Netherlands. Tel. $+31-53-892751$. sence of demagnetisation effects in the oriented planar direction, basic hysteretic and remanence measurements on the tape (or disc) can be used to analyse interaction effects, etc., and related to recording problems. However, this is not the case in perpendicular media. Making bulk magnetic measurements on samples in the perpendicular direction results in a large discrepency between the applied field and the internal field. This produces sheared hysteresis loops which must be corrected for demagnetisation if they are to be analysed in terms of the internal field. This is also true for remanence measurements. The usual technique of comparing the principle remanence curves using Henkel plots [1] and determining the interaction effects by comparison with the Wohlfarth relation [2] is only significant if remanence states are compared at the same internal field. It is therefore important to correct all measurements for the demagnetising field if the techniques used for analysis of longitudinal media are to be extended to perpendicular media.

In order to analyse the hysteretic properties of perpendicular media, it is often assumed that the demagnetising factor is close to that of a sheet (unity) and that the basic switching behaviour in 
terms of the internal field is a square loop. Shearing a square loop by the demagnetising effects produces a result which relates fairly closely to the observed behaviour [3] in many cases. In such systems, the shearing of the loop can be used to determine the magnetisation of the sample, provided that the demagnetisation factor is accurately known. The use of a sheet demagnetisation factor for thin film media is acceptable, but for media with a particulate nature, such as Alumite, this assumption may not be valid. "Striping out of domains" in some $\mathrm{Co}-\mathrm{Cr}$ samples can give rise to a rapid reduction of magnetisation in certain regions of the loop [4]. However, in many samples where magnetisation changes uniformly, shearing of a square loop seems satisfactory.

We have found that the converse is less satisfactory. By taking an experimental sheared loop and correcting for demagnetisation effects, on the assumption that the slope of the loop should be

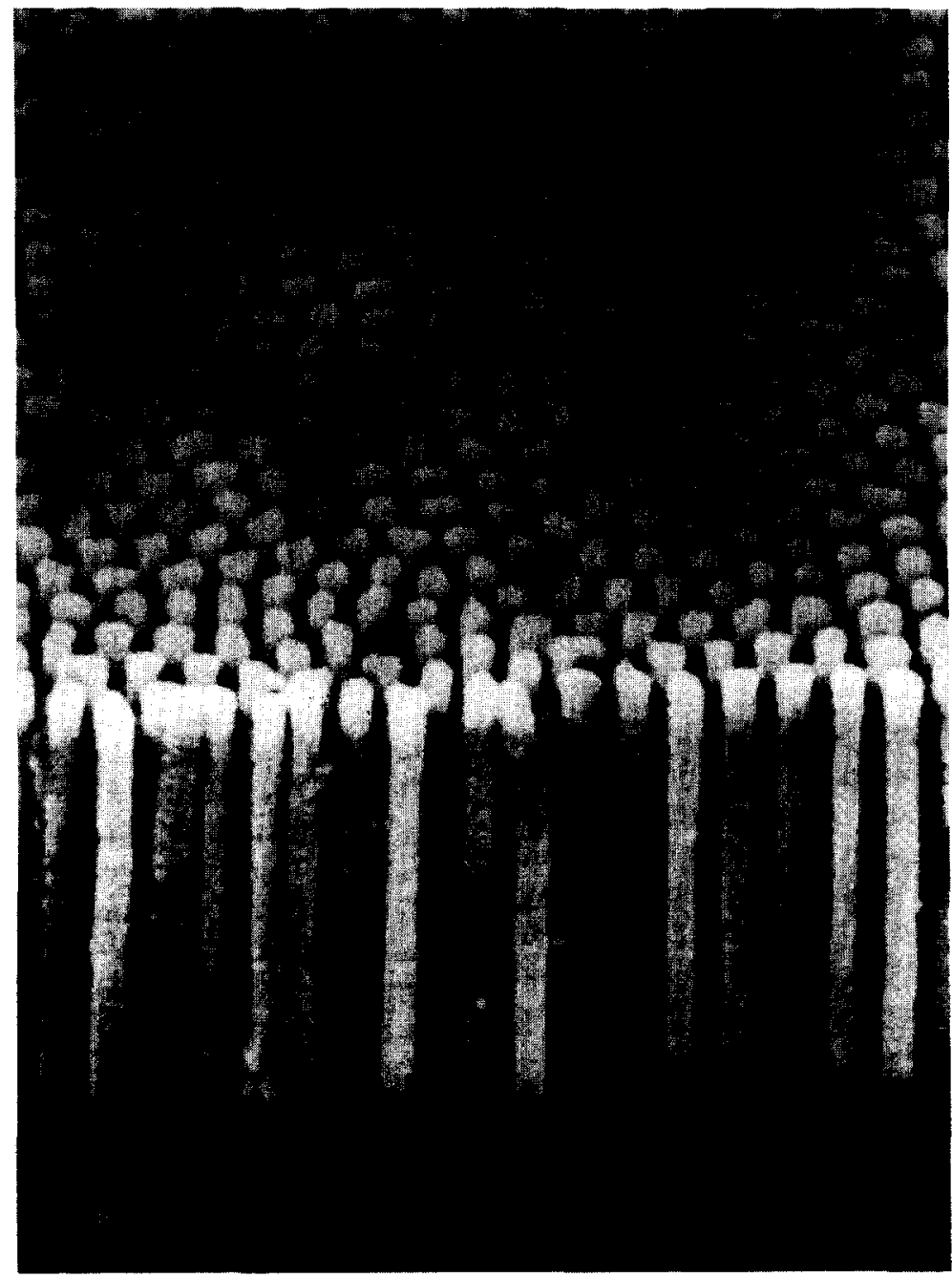

Fig. 1. Electron micrograph of an iron-filled Alumite sample ( $\left.t=600 \mathrm{~nm}, D_{\mathrm{p}}=45 \mathrm{~nm}, D_{\mathrm{c}}=78 \mathrm{~nm}\right)$ in which the $\mathrm{Al}_{2} \mathrm{O}_{3}$ matrix has been etched so that only the iron columns are visible. 
infinite at $H_{c}$, produces a loop whose exact shape is very sensitive to error in the determination of the correction factor. This is even more pronounced in remanence measurements [5] but is not unexpected, since $\mathrm{d} M / \mathrm{d} H$ is large in the corrected loop and small errors in the demagnetisation correction will be more evident.

Experimental investigation of the demagnetising effects is impossible because applicable measurement techniques measure the total moment of a sample and the shape of the hysteresis loop against internal field is unknown. We have therefore approached the problem by way of a model. Alumite has been chosen for this since it consists of thin magnetic columns in a regular hexagonal matrix which is relatively easy to model.

Alumite is produced on an aluminium disc which has been anodically oxidised. This results in pores perpendicular to the surface on a regular hexagonal array. These are chemically widened so that a magnetic material such as iron, cobalt or nickel can be deposited in the pores. By varying the preparation process, it is possible to vary the film thickness ( $t$ ), the pore (or magnetic column) diameter $\left(D_{\mathrm{p}}\right)$ and the cell size (or column spacing) $\left(D_{\mathrm{c}}\right)$. Electron microscopy on experimental samples indicates that pores are not always fully filled, there are defects in the hexagonal lattice and columns possess small lobes at the barrier layer next to the aluminium substrate (see fig. 1). However, for a model system, Alumite would seem ideal since it can be approximated to a regular lattice of needle-like particles.

\section{The model}

Experimental investigation of minor loops for Alumite systems indicate that reversible magnetisation changes play only a small part and hysteresis is dominated by irreversible processes [5]. It is therefore reasonable to assume that changes in magnetisation result from the irreversible switching of column moments and that, in the absence of interactions, the reversal of particles which are almost identical should produce a very narrow switching field. Thus, when corrected to internal field, the hysteresis loop should be square. Re- versible changes will, of course, produce rounding of the loop, but it can still be assumed that, in the region of the coercivity, $\mathrm{d} M / \mathrm{d} H$ should be infinite.

\subsection{Demagnetising field in a saturated sample}

The demagnetising field for a saturated Alumite sample can be modelled by considering the interaction energy between columns and the demagnetisation factor for individual columns. The demagnetising field can then be written as

$H_{\mathrm{d}}=\left(\gamma+N_{\mathrm{d}}\right) M$,

where $\gamma$ is an interal field parameter resulting from dipolar interactions between columns and $N_{\mathrm{d}}$ is the geometric demagnetisation factor along the column axis for an individual column. In the Alumite samples that we considered, the pore diameters $D_{\mathrm{p}}$ were all less than $45 \mathrm{~nm}$ and the film thickness was about $1000 \mathrm{~nm}$ so that, with an aspect ratio greater than $22, N_{\mathrm{d}}$ could be ignored. Ishii and Sato [6] calculated values for $\gamma$ by considering the magnetostatic energy $\epsilon_{R}$ between two parallel dipoles of length $t$ and spacing $R$ as

$\epsilon_{R}=\begin{aligned} & m^{2} \\ & 2 \pi\end{aligned}\left[\begin{array}{ll}\frac{1}{R} & \frac{1}{\sqrt{R^{2}+t^{2}}}\end{array}\right]$,

where $m$ is the pole strength. This is equivalent to one dipole producing a mean field $H_{R}$ at the other dipole given by

$H_{R}=\frac{m}{2 \pi t}\left[\frac{1}{R}-\frac{1}{\sqrt{R^{2}+t^{2}}}\right]$.

By considering Alumite as consisting of a hexagonal array of parallel dipoles, they were able to write an expression for the mean internal field at a single column as

$$
\begin{aligned}
H_{\mathrm{m}}= & \frac{3 m}{\pi t} \sum_{i=1}^{\infty} \sum_{j=0}^{i-1}\left[\frac{1}{\sqrt{i^{2}-i j+j^{2}}}\right. \\
& \left.-\frac{1}{\sqrt{i^{2}-i j+\left(t / D_{\mathrm{c}}\right)^{2}}}\right],
\end{aligned}
$$

where the values of $i$ and $j$ refer to fig. 2 and the 
sum is over $60^{\circ}$ section of the matrix and then multiplied by a factor 6 to give the total field. Equation (4) can then be expressed in terms of $M$, the mean magnetisation of the film, where

$M=\frac{2 m}{\sqrt{3}\left(D_{\mathrm{c}}\right)^{2}}$

and related to $H_{\mathrm{III}}$ by

$H_{\mathrm{m}}=\gamma M$,

resulting in an expression for $\gamma$ as

$$
\begin{aligned}
\gamma= & \frac{3 \sqrt{3}}{2 \pi\left(t / D_{\mathrm{c}}\right)} \sum_{i=0}^{\infty} \sum_{j=0}^{i-1}\left[\frac{1}{\sqrt{i^{2}+j^{2}-i j}}\right. \\
& \left.-\frac{1}{\sqrt{i^{2}+j^{2}-i j+\left(t / D_{\mathrm{c}}\right)^{2}}}\right] .
\end{aligned}
$$

This expression differs from that given in the paper by Ishii and Sato [5] since they worked in terms of mean magnetisation within columns which were initially assumed to be cylindrical. However, in converting back to mean magnetisation of the film, they omitted the cylindrical term and also ignored the fact that the hexagonal axes are at $60^{\circ} \mathrm{C}$ and not orthogonal, which produced a parameter $2 \sqrt{3} / \pi$ greater than our value.

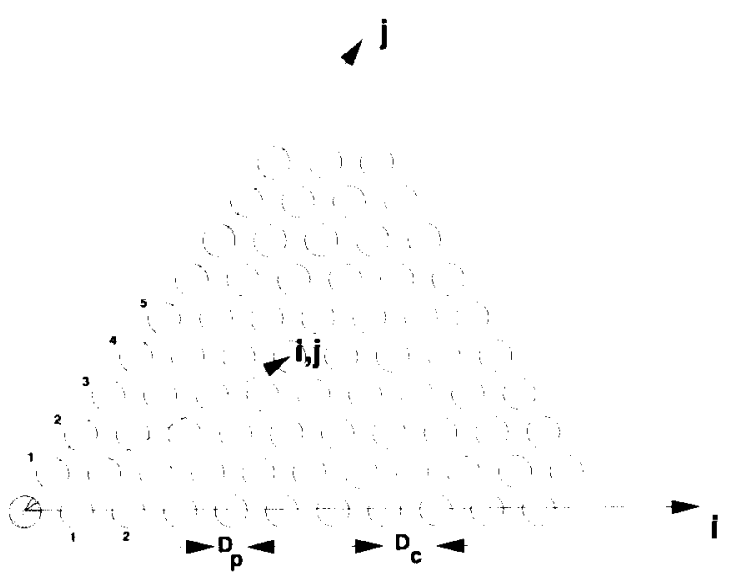

Fig. 2. Location of the columns used in calculating the internal field parameter at the shaded column. Only $\frac{1}{6}$ of the lattice needs to be considered due to the symmetry.

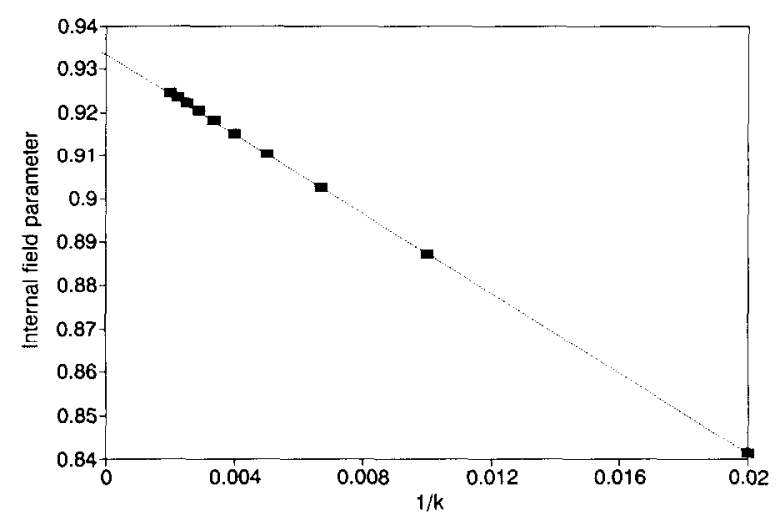

Fig. 3. The internal field parameter $\gamma$ against $1 / k$, where $k$ is the upper limit of the summation. Extrapolation back to $1 / k=0$ gives the true summation of eq. (5). The calculation is for a sample where $t / D_{c}=8.55$ (which corresponds to the sample shown in fig. 5).

The summation of eq. (5) has to be solved numerically. One technique is to numerically sum to a large value of $i$ and then use an approximate integral from there to infinity. However, this introduces an error which is dependent upon the column spacing and causes the value for $\gamma$ to be underestimated as the column spacing decreases. In eq. (5), if the upper limit of $\infty$ for the sum over $i$ is replaced by $k$, then, for large $k$, the sum approximates to a standard dipole term which goes as $(1 / \text { distance })^{3}$ with a dipole density which goes as (distance) $)^{2}$. Thus, the summation terms can be expected to go as $1 / k$ and a plot of $\gamma$ against $1 / k$ will be a straight line which can be extrapolated to $1 / k=0$ to give the true summation to $i=\infty$, as shown in fig. 3. By making this summation for a range of column spacings, a plot of $\gamma$ against $\ln \left(t / D_{c}\right)$ can be made, as shown in fig. 4. It can be seen that $\gamma$ approaches unity for large values of $\left(t / D_{c}\right)$, which is physically reasonable since this corresponds to closely packed columns when $\gamma$ should approach the sheet demagnetising factor.

The model assumes that columns can be regarded as line dipoles. This will lead to an error in the dipolar energy term when the assumption that $D_{\mathrm{c}} \gg D_{\mathrm{p}}$ is not valid. Calculations of this correction showed that the error was insignificant. In the Alumite sample with the largest 


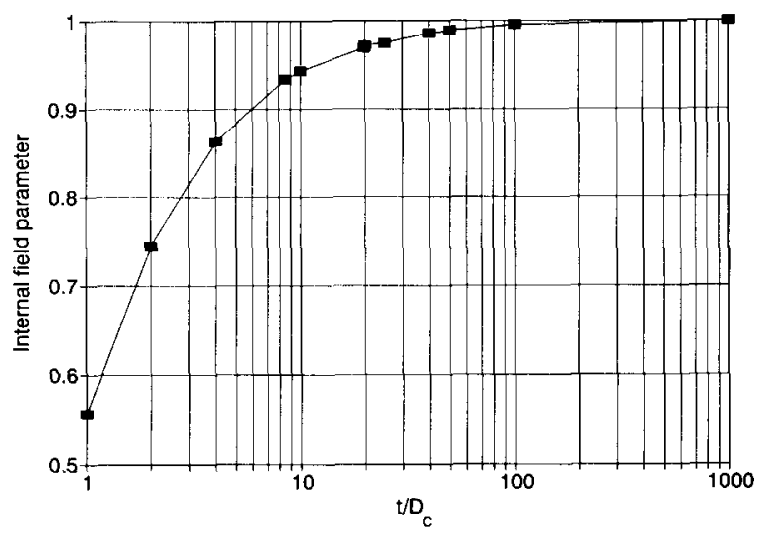

Fig. 4. Variation of the internal field parameter $\gamma$ with $\ln \left(t / D_{c}\right)$ showing an approach to unity as the structure approaches that of a continuous sheet.

value of $D_{\mathrm{p}}(72 \mathrm{~nm})$ and smallest $D_{\mathrm{c}}(117 \mathrm{~nm})$ an error of $2 \%$ was introduced into the term for adjacent columns. However, in the summation to infinity, this error became insignificant in the total value for $\gamma$.

The calculated value for gamma may then be used to shear a square loop and compare it with an experimental hysteresis loop to determine the magnetisation of the sample. Figure 5 shows such a determination for an iron-filled Alumite sample $\left(D_{\mathrm{c}}=117 \mathrm{~nm}, D_{\mathrm{p}}=45.0 \mathrm{~nm}\right.$ and $\left.t=1000 \mathrm{~nm}\right)$ with a calculated value for $\gamma=0.934$. Construc-

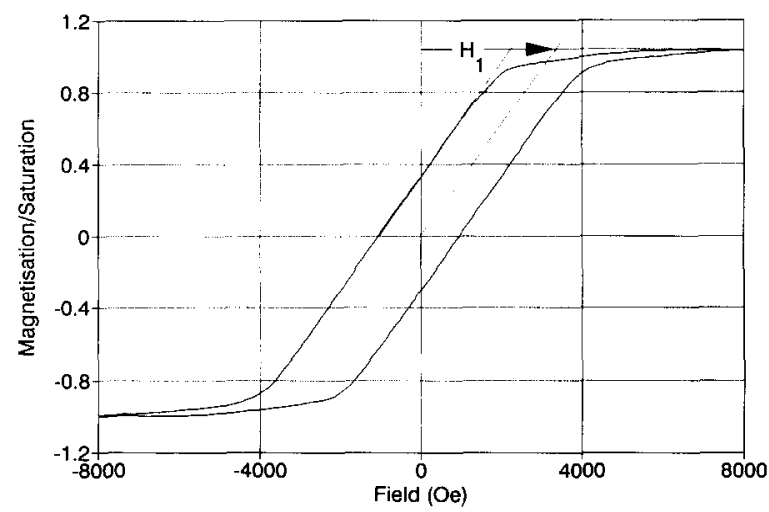

Fig. 5. A sheared hysteresis loop for an iron-filled Alumite sample $\left(D_{\mathrm{c}}=117 \mathrm{~nm}, D_{\mathrm{p}}=45 \mathrm{~nm}\right.$ and $\left.t=1000 \mathrm{~nm}\right)$ showing the calculation of its magnetisation from the value of $H_{1}$. Using the calculated value for $\gamma$ of 0.934 gives a saturation magnetisation $M_{\mathrm{s}}=3.60 \mathrm{kG}\left(286 \mathrm{~A} \mathrm{~m}^{-1}\right)$. tion of a line through the origin parallel to the hysteresis loop at $H_{\mathrm{c}}$ intersects the projection of the saturation magnetisation at $H_{1}$, where

$M_{\mathrm{s}}=\gamma H_{1} / \gamma$.

For the sample shown, this gives a measured value of $H_{1}=3.36 \mathrm{kOe}$ and saturation magnetisation $M_{\mathrm{s}}=3.60 \mathrm{kG}\left(286 \mathrm{k} \mathrm{Am}^{-1}\right)$. This is greater than the value determined by assuming the sheet demagnetisation factor as $7.5 \%$.

\subsection{Demagnetising field in a partly demagnetised sample}

In the derivation of a demagnetising field, it is assumed that the magnetisation is uniform throughout the sample, which results in a factor dependent only on geometry. However, in the case of an Alumite sample, as a reverse field is applied, it is expected that, apart from very small reversible changes in the magnetisation at the ends of the columns associated with the onset of a curling type reversal [7], a reduction in the total magnetisation of the sample will result from the irreversible magnetisation switching of individual columns. A calculation of the internal field in terms of a uniform sample magnetisation is therefore inappropriate. Furthermore, the distribution of the reversed columns will also have a bearing on the way $\gamma$ changes with magnetisation, i.e., whether there is cooperative behaviour or whether columns behave separately.

Consider a column which has reached its switching field. This field is the sum of the applied field and the interaction field due to the surrounding columns. If an adjacent column were to switch, the total field at the column would be reduced, owing to the reduction of the magnetostatic energy associated with a flux closure, and a column moment reversal would be less likely. This is essentially due to the lack of exchange coupling between the columns. Thus, we can assume that in Alumite cooperative switching is unlikely to be observed and columns will reverse in such a way as to keep reversed columns as widely spaced as possible. We have incorporated this into our model by considering a partly de- 
magnetised sample as consisting of the superposition of two uniformly magnetised matrices. One matrix has the original spacing $\left(D_{\mathrm{c}}\right)$ and is magnetised in the original field direction. The second matrix consists of columns with double the magnetisation in the reverse direction and has a wider spacing $D_{\mathrm{c}} S$, where $S$ is an integer and $S \geq 2$. The magnetisation can then be written as

$M=M_{\mathrm{s}}\left(1-\frac{2}{S^{2}}\right)$

and the expression for the demagnetisation field $H_{\mathrm{d}}$ as

$H_{\mathrm{d}}=\left(\gamma_{1}-\frac{2 \gamma_{2}}{S^{2}}\right) M_{\mathrm{s}}$,

where $\gamma_{1}$ and $\gamma_{2}$ are the internal field parameters for the matrices with spacings $D_{\mathrm{c}}$ and $D_{\mathrm{c}} S$, respectively, and $M_{\mathrm{s}}$ is the saturation magnetisation of the film.

Solution of eqs. (6) and (7) for integer values of $S$ from 2 upwards gives a value of $\gamma\left(=H_{\mathrm{d}} / M\right)$ which is magnetisation dependent and decreases as the magnetisation approaches saturation, as shown in fig. 6 .

The model assumes that the reversed columns lie on a matrix with integer spacing of $D_{c}$. This only allows calculations of $\gamma$ down to magnetisations of $0.5 M_{\mathrm{s}}$. However, if one assumes that for lower magnetisations the separation between the

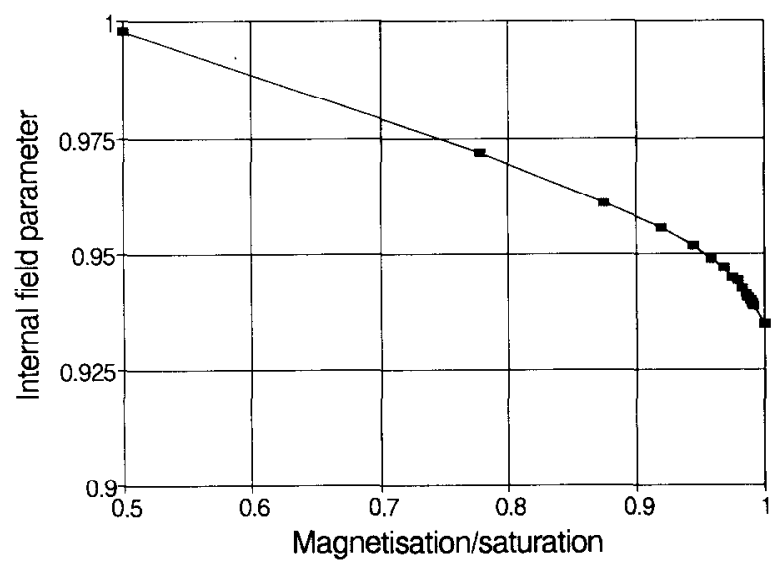

Fig. 6. The internal field parameter, $\gamma$, showing its variation with magnetisation as predicted by the model. The calculations are for the parameters of the sample shown in fig. 3 . reversed columns is maximised and, on average, the spacing of the reversed matrix can be a noninteger value of $S$ less than 2, then values for $\gamma$ can be calculated down to zero magnetisation.

\section{Conclusions}

(1) A model of the internal field of an Alumite film in terms of the interaction between columns produces an internal field parameter, $\gamma$, (equivalent to a demagnetising factor) which is less than the sheet demagnetisation factor.

(2) The value for $\gamma$ increases as the cell size is decreased and approaches unity for high density. This is physically reasonable since, for high densities, the system will approximate to a continuous sheet.

(3) The value for $y$ at saturation can be used to measure the demagnetisation of an Alumite sample for the sheared hysteresis loop.

(4) By extending the model to a partially magnetised sample, it is shown that the internal field parameter is magnetisation dependent.

\section{Acknowledgements}

This work was carried out within the framework of the EC Community Action on Magnetic Storage Technology (CAMST) and one of us (ES) is grateful to CAMST for financial support. We would also like to thank Y. Wakui of the Yamaha Research and Development Centre for providing the Alumite samples.

\section{References}

[1] G.W.D. Spratt, P.R. Bissell, R.W. Chantrell and E.P. Wohlfarth, J. Magn. Magn. Mater. 75 (1988) 309.

[2] E.P. Wohlfarth, J. Appl. Phys. 29 (1958) 595.

[3] M. Masuda and S. Shiomi, J. Magn. Jpn. 12 (1988) 537.

[4] J.C. Lodder, D. Wind, Th.J.A. Popma and A. Hubert, IEEE Trans. Magn. MAG-23 (1987) 2055.

[5] E.O. Samwel, P.R. Bissell and J.C. Lodder, J. Appl. Phys. (accepted).

[6] Y. Ishi and M. Sato, J. Magn. Magn. Mater. 82 (1989) 309.

[7] G.T. Huysmans, J.C. Lodder and J. Wakui, J. Appl. Phys. 64 (1988) 2016. 